Chapter 9

\title{
Multiplexing-Capable Immunobiosensor Based on Competitive Immunoassay
}

\author{
Yanfei Wang, Mingqiang Zou, Ping Yao and \\ Yuande Xu \\ Additional information is available at the end of the chapter \\ http://dx.doi.org/10.5772/52486
}

\section{Introduction}

As biological receptors, biosensors offer enormous potential for detecting a wide range of analytes in the food industry and in drug residue monitoring. For immunobiosensors, a probe is required to conjugate with a protein to monitor a specific interaction, and these probes possess reactive functionalities convenient for covalent linkage to antibodies and other protein molecules. As the usage of these probes becomes standardized, an increasing number of instruments have become widely available for measuring their signals. The development of multiplexing-capable immunobiosensors offers the possibility to simultaneously measure many different analytes in a small sample volume. The advantages of a multiplexing-capable immunobiosensor increase with each additional analyte analyzed and can be translated into substantial savings in the cost of reagents and time.

In this chapter, we review the main applications of multiplexing-capable immunobiosensors and illustrate their multiplexing capabilities by example of a suspension array system. First, the principle of a suspension array system is introduced. Microspheres are the common carrier in suspension array systems. Microspheres with different sizes or colors or a single microsphere with different fluorescent probes are used for multiplexed immunoassays (IAs).

Second, we list the main applications of suspension array systems. Suspension array technology was initially widely used in nucleic acid detection and genotyping. Recently, it has been gradually introduced for the detection and quantification of many viruses and antibodies in various samples based on IAs. However, there are also quite a few reports on multiplexed detection of low molecular weight compounds, such as drug residues, because this is 
more difficult for competitive IAs than for noncompetitive (sandwich) ones to meet the sensitivity and specificity requirements.

In the following section, a rapid, sensitive, multiplex, and competitive IA model based on a suspension array system is described. Antigens are covalently bound to different coded functional carriers to compete for antibodies with analytes in sample, and the fluorescent probe is used as a transducer. As the fluorescence signals are measured, the analytes can be screened simultaneously.

\section{Multiplexing-capable suspension array system}

\subsection{History of high-throughput technology}

High-throughput screening (HTS) has been developed as an advanced technique to perform large-scale screening of samples to analyze biological or chemical activity (Burbaum \& Sigal, 1997; Hill, 1998). Descriptions of this technology first appeared in the scientific literature as early as the 1980s. Over the past 30 years, the developments in the scale, efficiency, and technical level of HTS have rendered it a key technology in the areas of international drug research, genomics, proteomics, and analytical techniques (Burbaum, 1998; Eggeling et al., 2003; Haber et al., 2005).

HTS technology can be used to construct a micro-biochemical analysis system by integrating micromachining technology with microelectronics technology. With a large number of nucleic acid fragments with specific sequence or proteins fixed on a functional carrier, as well as labeled reactants (nucleic acids or protein) reacting in the reaction system, HTS can quantitatively determine the target analytes in samples by detecting the intensity of fluorescent reporter signals and can screen for compounds, nucleic acids, proteins, cells, and other biological components. Multiplexing and miniaturization are current trends in the development of HTS technology. Assays performed on large numbers of candidates in small sample volumes and using target analytes (e.g., antigens, antibodies, nucleotides, and peptides) can be screened. HTS has important application prospects in the fields of drug discovery, drug screening, and diagnostics, and suspension array technology is one of the representatives in a variety of new high-throughput analysis methods.

\subsection{Suspension array system and its detection principles}

The suspension array system is based on encoded microspheres, which are both the encoded carrier and analysis platform for the probes. Compared with traditional flat biochips, the advantages of suspension biochips are as follows (Meza, 2000; Battersby, 2000; Grondahl, 2000):

- Liquid phase conditions are conducive for maintaining the natural conformation of protein molecules, and the use of microspheres as the solid carrier in the assay leads to reaction kinetics approaching phase conditions; 
- Differently coded microspheres conjugated to the capture antibody or target molecules are required for each reaction and can be flexibly pooled together for a multiplexed assay and separated later during data acquisition, reducing the analysis cost;

- These "no-wash" assays eliminate the need for washing steps; and

- Suspension array systems offer an approach for large-scale screening, which is an important breakthrough in the number of analytes compared with traditional flat chips.

Of course, suspension array system has some shortcomings: optimization of multiple liquid phase reaction conditions is more difficult, and the cross reaction and its elimination is more complicated which would require thorough analysis. In addition, on-line monitoring of analytes could not be done using suspension array system.

In spite of this, with widespread prospects for application,suspension array technology is becoming a research focus.

Microsphere-based suspension array technology consists of a detection platform and microspheres. Such technology has a long history of simplifying the separation of biological systems, the positioning of biological interactions, and signal amplification in the biomedical field (Brown, 1987; Deleo, 1991). After decades of development, microsphere-based analysis techniques have now been developed into a wide range of analysis systems to meet specific needs. However, despite their various forms, the basic principle of these assays is microspheres pre-encoded by chemical, spectral, electronic, or physical methods. Each microsphere encodes specific identifying information, and these microspheres offer binding sites for bioconjugation. Before testing, differentially coded microspheres are coupled with the capture antibody or target molecules (e.g., antigens, oligonucleotides, receptors, or peptides) that are required for each reaction. Then, a reporter molecule labeled with a fluorescent marker (e.g., phycoerythrin or Alexa 532) binds to the analytes captured on the microspheres in the sample solution. The bound microspheres are passed through the detection channel and separated during data acquisition.

It can be concluded that microspheres-based suspension array technology includes two key factors: 1) coding and decoding a large number of microspheres, and 2) conjugating capture antibodies or target molecules with functional microspheres for the identification of different analytes.

As a typical multiplexed microsphere-based suspension array platform, the Luminex ${ }^{\circledR}$ XMAPTM system is a flow-based dual-laser system capable of analyzing and reporting up to 100 different analytes in a single reaction vessel in just a few seconds per sample (Dubar, 2006).The target molecules are fixed on the carrier via non-covalent or covalent bonds. Usually, reactive functionalities on microspheres provide the means to specifically label certain target groups on capture antibodies or target molecules in the form of a covalent bond. The common covalent bonds include amide, ester, and ether bonds (Deleo, et al. 2000; Mehnaaz, et al. 2003). 


\section{Competitive model based on IA}

\subsection{Immunoassay (IA)}

Immunoassay technology is a type of analysis method based on antigen-antibody specificity, identification, and binding. Depending on the available antigen or antibody reagents, one can achieve qualitative and quantitative detection of a specific analyte.

Immunoassay is a very popular method, has been rapidly developed by many research laboratories, and many versions are commercially available due to their rapid, simple, specific detection capabilities. Different types ofIA with specific characteristics listed in Table 1 would give a comparison. In the early 1990s, IA, together with liquid chromatography (LC) and gas chromatography (GC), were called three of the most advanced analytical techniques by the Association of small molecule residue determination mainly uses competitive IA.

\begin{tabular}{ll}
\hline \multicolumn{1}{c}{ type } & \multicolumn{1}{c}{ characteristics } \\
\hline Radioimmunoassay, RIA & $\begin{array}{l}\text { Reagent with low cost, high sensitivity; complex operation, radioactive } \\
\text { pollution, short duration }\end{array}$ \\
\hline enzyme immunoassay, EIA & $\begin{array}{l}\text { Reagent with low cost, simple operation; low sensitivity, suitable for } \\
\text { qualitative and semi-quantitative determination }\end{array}$ \\
\hline $\begin{array}{l}\text { chemiluminescent enzyme } \\
\text { immunoassay,CIZIA }\end{array}$ & $\begin{array}{l}\text { Simple operation, low cost, high sensitivity, stable reagent; working curve } \\
\text { drift with time }\end{array}$ \\
\hline fluorescence immunoassay,FIA & $\begin{array}{l}\text { High sensitivity, stable reagent; complex operation, high cost of reagents, } \\
\text { the background interference }\end{array}$ \\
\hline
\end{tabular}

Table 1. Different types oflA with specific characteristics

\subsection{Operational process of competitive IA}

The basic operational process of the competitive model includes fixing the antigen on a solid support, pooling the sample (antigen) and antibody in the reaction vessel together for competitive interaction, and adding a reporter molecule labeled with a marker to the sample solution to bind to the analyte captured by the antigen fixed on the solid carrier. Competitive IA can quantitatively determine the analyte in a sample indirectly by detecting the intensity of the reporter signals because the detection signal is inversely proportional to the concentration of the analyte in a competitive model. Small molecular weight compounds, such as drug residues, and theconsist of preparation of the immunogen (artificial antigen), production of the antibody, and optimization of reaction conditions. 
Small molecular weight compounds enzyme-linked immunosorbent assay became an inevitable trend, and multiplexed assays have been further simplified by introducing immunosensors, which are available for HTS and automation. Remarkably, optical immunosensors are widely employed to their full potential.

\section{Applications of a suspension array system based on competitive IA}

\subsection{Main applications of a suspension array system}

Microsphere-based suspension array technologies, such as the Luminex ${ }^{\circledR}$ xMAPTM system, offer a flexible technical platform based on Flexible Multi-Analyte Profiling (xMAP) technology, which was recently developed (Morgan, et al. 2004). Such technology combines encoded microspheres with flow cytometry. Two lasers analyze the microspheres in a flow stream. The first laser indentifies each microsphere-associated analyte according to the fluorescent signature of the microsphere, and the second measures the reporter molecules attached to the analytes quantify the amount of analyte (Nolan \&Sklar, 2002).

Many studies have been performed in the field of clinical diagnosis and genomic research by combining flow-based technology with IA (Yan et al. 2004; Fuja et al. 2004). Beads of different sizes/colors or a single bead with different fluorescent probes are used for multiplexed IA. Previous studies using multiplexed assay technology include detection of thyroxine and thyrotropin from blood spot samples for congenital hypothyroidism (Bellisario, et al. 2000), antibodies to West Nile virus in human serum and cerebrospinalfluid (Wong et al. 2004), and Ig classes in serum and stool samples (Dasso, et al. 2002), as well as identifying response patterns in hyperinflammatory diseases (Hsu, et al. 2008) and cytokines in human serum (Ray et al. 2005). As an open platform, xMAP was gradually introduced for the detection and quantification of many virus and drug residues in food and agriculture samples. For example, a multiplex microsphere IA was developed to monitor seed potatoes for potato viruses $X, Y$, and PLRV (Bergervoet et al. 2008), and a flow cytometric IA was developed to detect sulfonamide residues in milk (de Keizer et al. 2008).

Notably, the multiplexed IAs using suspension array technology are currently more prevalent for analyzing viruses and antibodies than detecting small molecular weight compounds such as drug residues.

A recent study reports the use of a suspension array system for the detection of drug residues, highlighting its simplicity, low cost, specificity, and multiplexing capabilities (Zou et al. 2008). Fig. 1 shows the competitive IA strategy and the detection principles of the Luminex® ${ }^{\circledR M A P T M}$ system. 


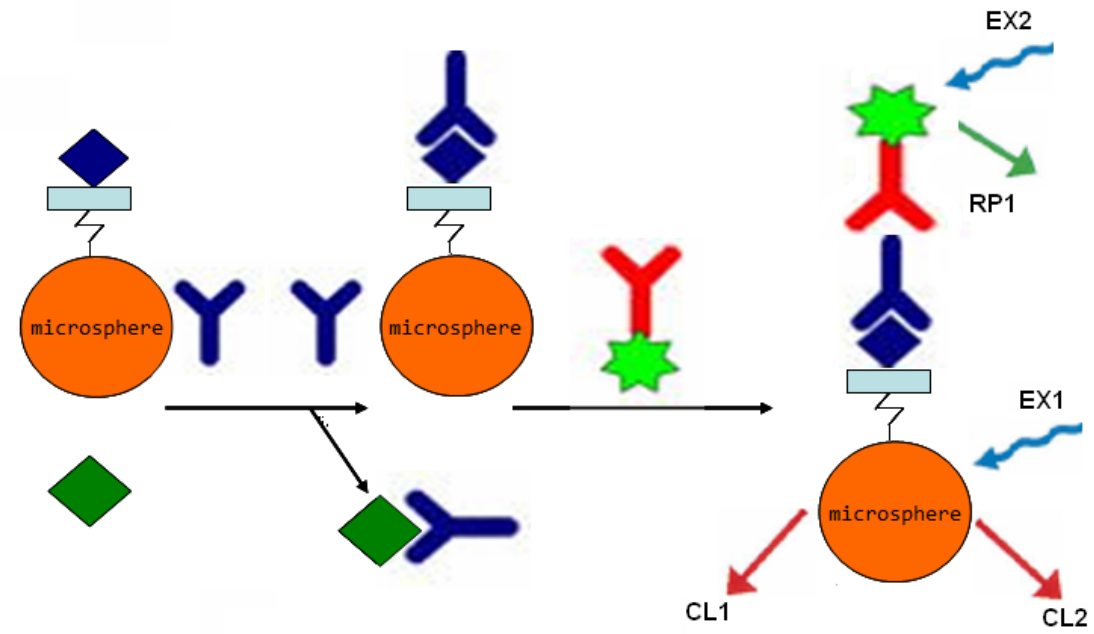

Figure 1. Microsphere-based competitive IA strategy. EX: excitation laser; RP: reporter laser; CL: clarification laser

\subsection{Detection of low molecular weight compounds using an immunobiosensor based on competitive IA}

Low molecular weight compounds are widely utilized as animal drugs, food additives, and pesticides to pursue maximum productivity and profits directly or indirectly in food products. However, residues of such low molecular weight compounds in food products are detrimental to human health. For instance, clenbuterol (313.65u), a $\beta$-adrenergic agonist growth promoter, is associated with a series of severe food poisoning outbreaks around the world. Therefore, quantitative analysis of such low molecular weight compounds is essential to safeguardpublic health, prevent their illicit use, and facilitate governmental regulation and surveillance.

In comparison to conventional ELISAs, a microsphere-based competitive fluorescent IA (MCFI) has been established to analyze low molecular weight compounds in food as described in the literature (Zou et al. 2008). This method utilizes an artificial antigen bound to 4$\mu \mathrm{m}$ polystyrene beads together with an FITC-conjugated monoclonal antibody. In this indirect IA, the fluorescent conjugated antibody is bound to an artificial antigen fixed on the polystyrene beads and subsequently analyzed by multiparameter flow cytometry. Quantification is based on the intensity of the fluorescent reporter signal: the higher the hazardous low molecular weight compound concentration, the lower the fluorescence intensity.

It is worth pointing out that the entire process is specially designed for aqueous systems that are bio-compatible with proteins' native structures. To illustrate the feasibility of this novel strategy, clenbuterol was selected as a practical analyte. Compared to traditional IAs, such as ELISA, microsphere-based competitive fluorescent IA by flow cytometry offers comparable or higher sensitivity, better reproducibility, and a greater dynamic range. 


\subsection{Simultaneous detection of antibiotics in raw milk}

Antibiotics, as conventional veterinary medicine, are widely used for the treatment and prevention of microbial infections. Antibiotic residues can enter the body and are metabolized by the liver. Further, antibiotics and their metabolites can impair the nervous and psychiatric systems, circulatory system, and even brain function before elimination by the kidneys. Thus, every country has established a maximum residue limit (MRL) for the total amount of antibiotics in various food products (e.g., raw milk) to bolster public health and the security of international trade in food products. For example, the European Union established a MRL of $100 \mu \mathrm{g} / \mathrm{L}$ for the total amount of gentamycin in milk and, $150 \mu \mathrm{g} / \mathrm{L}$ for the total amount of kanamycin in milk.

As previously published (Wang, et al. 2011), we applied suspension array technology to develop an indirect multiplexed competitive IA for simultaneous detection of antibiotic residues, such as kanamycin and gentamycin, in raw milk. First, we successfully produced a monoclonal antibody $(\mathrm{McAb})$ against gentamycin. Then, with the coating antigens of kanamycin and gentamycin attached to differentially encoded beads and R-phycoerythrin-conjugated goat anti-mouse IgG as the fluorescent probe, an indirect bead-based competitive fluorescent IA using a Bio-Plex ${ }^{\mathrm{TM}} 200$ suspension array system was developed. To develop the actual application, raw milk samples spiked with kanamycin or gentamycin were analyzed using ELISA and our Bio-Plex ${ }^{\mathrm{TM}}$ assay for comparison.

$\mathrm{A} \mathrm{McAb}$ against gentamycin was successfully obtained, and an indirect bead-based competitive fluorescent IA was developed for rapid detection of small molecule drug residues in animal-derived food products, such as raw milk. Notably, even with the matrix's background interaction with milk, the fluorescent intensities detected from raw milk samples are still strong enough and sufficient to detect the lowest allowable quantity of the antibiotics in milk at the MRL level. In fact, cephalosporins and sulfonamides, which are used worldwide in cows and are also tested in milk, could be added to the assay because the suspension array technology permits up to 100 different assay combinations. Compared to ELISA, the multiplexed assay had a similar limit of detection,but its ability to simultaneously measure several analytes in a single sample is superior to ELISA. Details of limit of detection, detection time, etc. are described in the literature (Wang, et al. 2011).

With a particular focus on applications in real samples containing complex matrices, kanamycin was intraperitoneally administered to three cows at a dose of $10 \mathrm{mg} / \mathrm{kg}$ (twice per day for 3 days), and gentamycin was administered in another three cows in the same way.

Sixty raw milk samples were collected from these six cows. Fig. 2 and Fig. 3 show the comparison of the detection results obtained by the bead-based competitive fluorescent IA and ELISA. The analysis shows that there is good correlation between the results of the multiplexed assay and those of ELISA. Basically, the kanamycin concentrations in raw milk decreased gradually. Seven hours after the last injection, the residues were present at 190 $\mathrm{ng} / \mathrm{ml}$, and $20 \mathrm{~h}$ later, the residues were reduced to $40 \mathrm{ng} / \mathrm{ml}$, which is lower than the MRL provided by the European Union. The gentamycin concentrations in raw milk decreased in a similar manner. 

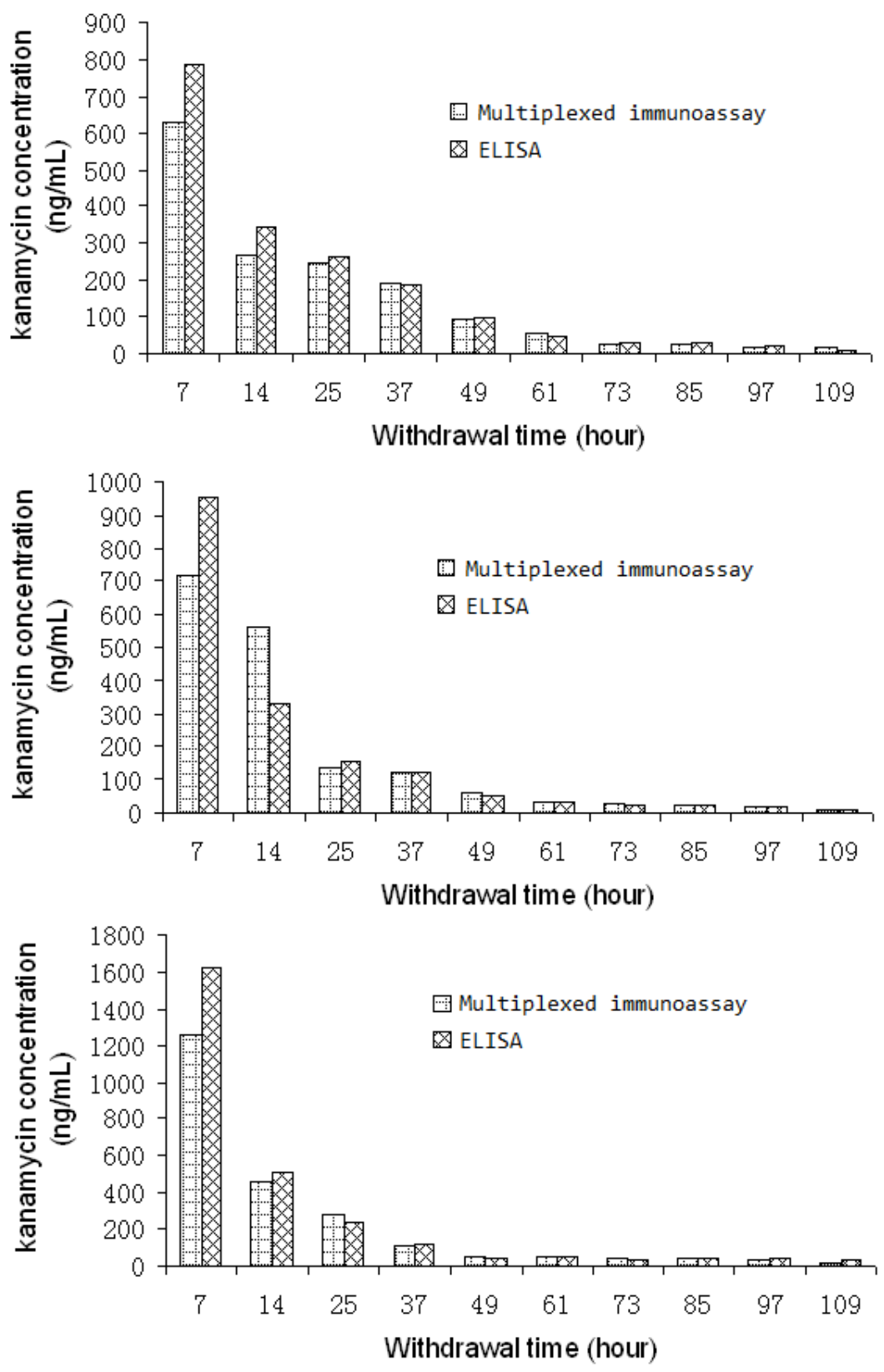

Figure 2. Raw milk depletion profile of kanamycin after intramuscular administration to cows. 

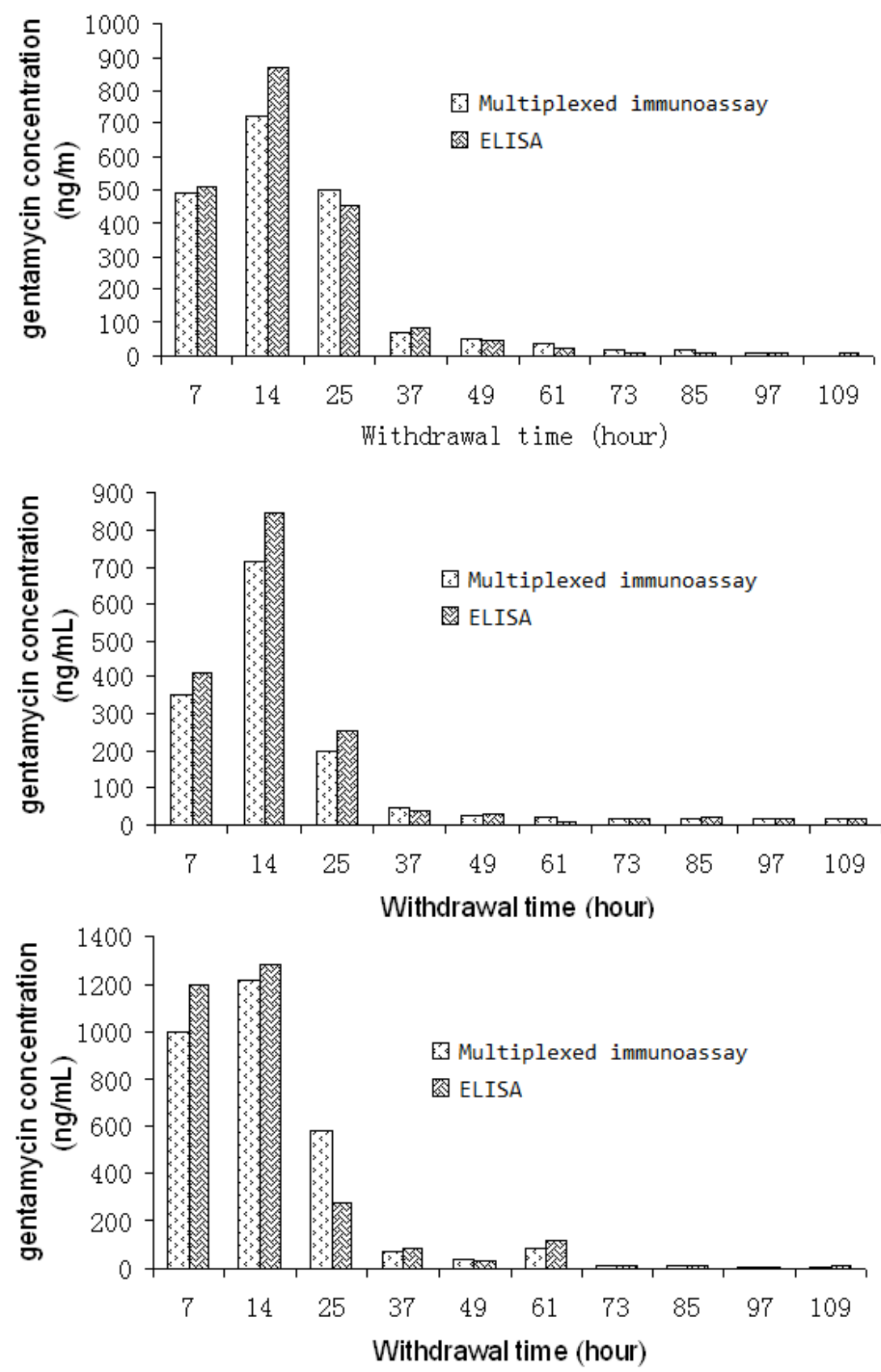

Figure 3. Raw milk depletion profile of gentamycin after intramuscular administrationto cows. 


\subsection{Other applications using immunobiosensors based on competitive IA}

To illustrate the feasibility of this competitive strategy, we present an example of the simultaneous detection of antibiotic residues like kanamycin and gentamycin, though other related reports exist.

Sulfonamides are a class of antibiotics used in veterinary and human medicine for the treatment and prevention of microbial infections. The European Union (EU) established a MRL of $100 \mu \mathrm{g} / \mathrm{L}$ for the total amount of sulfonamides in milk. To detect sulfonamides in raw milk, a multi-sulfonamide flow cytometric IA (FCI) was developed using the xMAP technology as described in the literature (de Keizer, et al. 2008). In this automated FCI, a previously developed biotinylated multi-sulfonamide mutant antibody (M.3.4) was applied in combination with fluorescent beads, directly coated with a sulfathiazole derivative, and streptavidin-phycoerythrin (SAPE) for detection. Because of differences in sensitivity toward different sulfonamides, the FCI was considered and validated as a qualitative screening assay.

Simultaneous detection for three kinds of veterinary drugs: chloramphenicol (CAP), clenbuterol, and 17-beta-estradiol has also been reported (Liu, et al. 2009). Conjugates of chloramphenicol and clenbuterol coupled with bovine serum albumin were synthesized and purified. Probes for the suspension array were created by coupling the three conjugates on fluorescent microspheres, and the microstructures on the microspheres' surfaces were observed by scanning electron microscopy, which was direct confirmation for successful conjugate coupling. The addition of conjugates and the amounts of antibodies were optimized and selected, respectively. The suspension array is specific and has no significant cross-reactivity with other chemicals. Meanwhile, unknown samples were analyzed by suspension array and ELISA in comparison with each other. The errors found for the detection of the unknown samples were relatively small in both methods, but the detection ranges of the suspension array are broader and more sensitive than that of traditional ELISA.

Further research investigated the simultaneous quantitative determination of five antibiotics (tylosin, tetracycline, gentamycin, streptomycin, and chloramphenicol) in milk in the same research laboratory (Su, et al. 2011). A novel treatment of milk samples for the suspension array with diethyl ether was performed, which greatly reduced the interference of the disturbing components in milk on the reaction results with no significant effect on detection sensitivity. Compared to using a biotin-labeled monoclonal antibody, using a secondary biotinylated antibody further increased the detection sensitivity. Thus, suspension assay technology is powerful for the rapid, quantitative analysis of multiple drug residues.

\section{Conclusions}

In this chapter, the competitive IA method with immunobiosensor as a detection platform was first used with a single analyte in simple buffer for its sensitivity, specificity, and repeatability, and then complex substrates such as milk were introduced. Thousands of actual 
samples were screened for confirmation of this multiplexing-capable immunobiosensor based on competitive IA. As an easy, effective, and time-saving method, the multiplexingcapable immunobiosensor based on competitive IA has the potential to detect small molecular weight compounds in various actual applications. Previous research has laid a firm foundation for simultaneous screening of multiple analytes in food products. Future research should focus on adding more analytes to the assay and establishing a reliable multiplexed assay to detect analytes in additional samples, such as tissue and fat. It is a flexible technical platform with multiplexing capabilities.

\section{Acknowledgments}

This work was partially supported by a Strategic Eleventh Five-Year Science and Technology Supporting Grant No: 2009BAK61B04 and Special Funded Projects of the Fundamental Research Funds from the Chinese Academy of Inspection and Quarantine of China No: 2010JK016.

\section{Author details}

Yanfei Wang ${ }^{1}$, Mingqiang Zou ${ }^{1}$, Ping $\mathrm{Yao}^{2}$ and Yuande $\mathrm{Xu}^{3}$

1 Chinese Academy of Inspection and Quarantine, China

2 The People's Hospital of Juxian, China

3 COFCO Corporation, China

\section{References}

[1] Burbaum, J. J., \& Sigal, N. H. (1997). New technologies for high-throughput screening.Curr.Opin. Chem. Biol. , 1, 72-78.

[2] Hill, D. C. (1998). Novel screen methodologies for identification of new microbial metabolites with pharmacological activity. Adv. Biochem. Eng. Biotechnol. , 59, 73-121.

[3] Burbaum, J. J. (1998). Miniaturization technologies in HTS: how fast, how small, how soon? Drug Discov. Today , 3, 313-322.

[4] Eggeling, C., Brand, L., Ullmann, D., et al., \& (2003, . (2003). Highly sensitive fluorescence detection technology currently available for HTS.Drug Discov.Today. , 8, 632-641. 
[5] Haber, C., Boillat, M. ., \& Schoot, B. V. D. (2005). Precisenanoliter fluid handling system with integrated high-speed flow sensor. Assay Drug Dev. Technol. , 3, 203-212.

[6] Meza, M. B. (2000). Bead-based HTS applications in drug discovery.Drug Discov.Today (Suppl.). , 5, 38-41.

[7] Battersby, B. J., Bryant, D., Meutermans, W., et al. (2000). Toward larger chemical libraries: encoding with fluorescent colloids in combinatorial chemistry. J. Am. Chem. Soc. , 122, 2138-2139.

[8] Grondahl, L. B., Bronwyn, J., Bryant, D., et al. (2000). Encoding combinatorial libraries: a 104novel application of fluorescent silica colloids. Langmuir. , 16, 9709-9715.

[9] Brown, W., \& (1987, . (1987). Microparticle-capture membranes: Application to Test Pack hCG-urine. Clin. Chem. , 33, 1567-1568.

[10] Deleo, D. T., \& (1991, . (1991). Particle-enhanced turbidimetric IA of sex-hormonebinding globulin in serum.Clin. Chem. , 37, 527-531.

[11] Jane, A., Ferguson, F. J., Walt, D. R., \& (2000, . (2000). High-density fiber-optic DNA random microsphere array.Anal. Chem. , 72, 5618-5624.

[12] Mehnaaz, F. A., Romy, K., Adrian, P. G., et al. (2003). DNA hybridization and discrimination of single-nucleotide mismatches using chip-based microbead arrays. Anal. Chem. , 75, 4732-4739.

[13] Bellisario, R., Colinas, R. J., Pass, K. A., \& (2000, . (2000). Simultaneous Measurement of Thyroxine and Thyrotropin from Newborn Dried Blood-Spot Specimens Using a Multiplexed Fluorescent Microsphere IA.Clini. Chem. , 46, 1422-1424.

[14] Bergervoet, J. H. W., Peters, J., van Beckhoven, J. R. C. M., van den, Bovenkamp. G. W., Jacobson, J. W., \& van der Wolf, J. M. (2008). Multiplex microsphere immuno-detection of potato virus Y, X and PLRV. J. Virol. Methods. , 149, 63-68.

[15] Dasso, J., Lee, J., Bach, H., Mage, R. G., \& (2002, . (2002). A comparison of ELISA and flow microsphere-based assays for quantification of immunoglobulins. J. Immunol. Methods. , 263, 23-33.

[16] de Keizer, W., Bienenmann-Ploum, M. E., Bergwerff, A. A., Haasnoot, ., \& , W. (2008). Flow cytometric IA for sulfonamides in raw milk. Anal.Chim.Acta. , 620, 142-149.

[17] Fuja, T., Hou, S., Bryant, P., \& (2004, . (2004). A multiplex microsphere bead assay for comparative RNA expression analysis using flow cytometry.J Biotechnol. , 108, 193-205.

[18] Hsu, H. Y., Wittemann, S., Schneider, E. M., Weiss, M., Joos, ., \& , T. O. (2008). Suspension microarrays for the identification of the response patterns in hyperinflammatory diseases. Med. Eng. Phys. , 30, 976-983.

[19] Liu, N., Su, P., Gao, Z. X., Zhu, M. X., Yang, Z. H., Pan, X. J., Fang, Y. J., \& Chao, F. H. (2009). Simultaneous detection for three kinds of veterinary drugs: Chloramphenicol, 
clenbuterol and 17-beta-estradiol by high-throughput suspension array technology. Anal.Chim.Acta. , 632, 128-134.

[20] Morgan, E., Varro, R., Sepulveda, H., Ember, J. A., Apgar, J., Wilson, J., Lowe, L., Chen, R., Shivraj, L., Agadir, A., Campos, R., Ernst, D., \& Gaur, A. (2004). Cytometric bead array: a multiplexed assay platform with applications in various areas of biology. Clin.Immunol. , 110, 252-266.

[21] Nolan, J. P. ., Sklar, L. A., \& (2002, . (2002). Suspension array technology_evolution of the flat-array paradigm.TRENDS Biotechnol. , 20, 9-12.

[22] Ray, C. A., Bowsher, R. R., Smith, W. C., Devanarayan, V., Willey, M. B., Brandt, J. T., \& Dean, R. A. (2005). Development, validati. on, and implementation of a multiplex IA for the simultaneous determination of five cytokines in human serum. J. Pharmaceut. Biomed. , 36, 1037-1044.

[23] Wong, S. J., Demarest, V. L., Boyle, R. H., Wang, T., Ledizet, M., Kar, K., Kramer, L. D., Fikrig, E., Koski, ., \& , R. A. (2004). Detection of Human Anti-Flavivirus Antibodies with a West Nile Virus Recombinant Antigen Microsphere IA. J. Clin. Microbiol. , $42,65-72$.

[24] Yan, X. M., Tang, A. J., Schielke, E. G., Hang, W., \& Nolan, J. P. (2004). On the development of a microsphere-based multiplexed IA for influenza virus typing and subtyping by flow cytometry. International Congress Series. , 1263, 342-345.

[25] Zou, M. Q., Gao, H. X., Li, J. F., Xu, F., Wang, L., \& Jiang, J. Z. (2008). Rapid determination of hazardous compounds in food based on a competitive fluorescence microsphere IA.Anal.Biochem. , 374, 318-324.

[26] Wang, Y. F., Wang, D. N., Zou, M. Q., Jin, Y., Yun, C. L., Gao, ., \& , X. W. (2011). Application of suspension array for simultaneous detection of antibiotic residues in raw milk.Analytical letters. , 44, 2711-2720.

[27] Dubar, S. A. (2006). Applications of Luminex ${ }^{\circledR}$ xMAP ${ }^{\mathrm{TM}}$ technology for rapid, highthroughput multiplexed nucleic acid detection. Clin.Chim.Acta. , 363, 71-82.

[28] Su, P., Liu, N., Zhu, M. X., Ning, B. A., Liu, M., Yang, Z. H., Pan, X. J., Gao, ., \& , Z. X. (2011). Simultaneous detection of five antibiotics in milk by high-throughput suspension array technology. Talanta. , 85, 1160-1165. 
